\title{
Opportunistic Infections among HIV/AIDS Patients taking Ante-Retroviral Therapy at Tertiary Care Hospital in Wolaita Zone, Southern Ethiopia
}

\section{Mihiretu Alemayehu*, Yilma Yisehak², Worku Alaro² and Bereket Alemayehu ${ }^{3}$}

${ }^{1}$ School of Public Health, College of Health Sciences and Medicine, Wolaita Sodo University, Wolaita Sodo, Ethiopia

${ }^{2}$ Wolaita Zone Health Department, Wolaita Sodo, South Nations, Nationalities and People's Regional state, Ethiopia

${ }^{3}$ Biomedical Science Division, Department of Biology, College of natural and computational science, Wolaita Sodo University, Wolaita Sodo, Ethiopia

\begin{abstract}
Introduction: Opportunistic infections, which mostly manifest at advanced stage of HIVIAIDS, are the leading causes of morbidity and mortality despite the existence of prevention and treatment modalities. People with advanced HIV infection are vulnerable to opportunistic infections and malignancies because of the opportunity offered by a weakened immune system. Since the types of opportunistic infections vary from region to region, for the strategies of morbidity and mortality to be reduced, identification of opportunistic infections, their frequency and distribution plays significant role.
\end{abstract}

Methodology: A facility based cross-sectional study was conducted from August - September, 2016 in Wolaita Sodo teaching and referral hospital, Southern Ethiopia, among HIVIAIDS patients who were taking ante-retroviral therapy with in the past five years preceding the survey. A total of 377 study participants were selected through systematic sampling technique and a pre-tested checklist was used to collect data from clinical records of study subjects. The data was entered and analyzed by SPSS version 20 .

Result: The overall magnitude of opportunistic infections was $45.3 \%$. Only $6.9 \%$ of them had co-infections. The most prevalent opportunistic infection was oral candidiasis (17.7\%), followed by herpes zoster (15.5\%) and pulmonary tuberculosis $(11.9 \%)$. The cumulative prevalence of all forms of tuberculosis, $(19.4 \%)$, was identified as the leading opportunistic infection. Cryptoccocal meningitis $(2.8 \%)$, cryptosporidiosis $(2.5 \%)$ and recurrent mycosis $(1.4 \%)$ were the least commonly observed opportunistic infections. AIDS defining illnesses such as CNS toxoplasmosis, Cryptoccocal meningitis, pneumocystis carini pneumonea and cryptosporidiosis with diarrhea, which commonly manifest during the end stage of HIVIAIDS, cumulatively account for $14.6 \%$ of opportunistic infections.

Conclusion: A high prevalence of opportunistic infection was identified among HIV/AIDS patients taking antiretroviral therapy. This indicates that the threatening burden of opportunistic infections couldn't be averted by sole provision of ante-retroviral therapy. Therefore, in order to reduce the magnitude of the diseases, strategies beyond providing ante-retroviral therapy are required. In addition, researchers and other stake holders have to conduct longitudinal studies in order to identify risk factors for respective opportunistic infections.

Keywords: Opportunistic infection; HIV/AIDS; Wolaita; WHO clinical stage

Abbreviations: AIDS: Acquired Immune-Deficiency Syndrome; ART: Ante-Retroviral Therapy; CNS: Central Nervous System; HIV: Human Immuno-Deficiency Virus; OI: Opportunistic Infection; PCP: Pneumocystis Carini Pneumonea; WHO: World Health Organization

\section{Introduction}

The clinical symptoms of HIV infection encompass a spectrum ranging from primary infection and prolonged non symptomatic state to advanced disease. Opportunistic infections, which mostly manifest at advanced stage of the disease, are the predominant causes of morbidity and mortality among HIV-infected patients. Despite the existence of prevention and treatment modalities, opportunistic infections continued to be the leading causes of morbidity and mortality among people living with HIV/AIDS [1]. Even though the number of people living with HIV on antiretroviral therapy has increased from 7.5 million in 2010 to 17.0 million in 2015, providing treatment didn't entirely take out the need for preventing opportunistic infections mostly because of poor adherence, exposure to potential pathogens, malnutrition, the degree of host immunity, psychological stress, life style and others factors [2].

People with advanced HIV infection are vulnerable to opportunistic infections and malignancies because of the opportunity offered by a weakened immune system due to progressive depletion of the CD4 $\mathrm{T}$ cells count. After an initial asymptomatic phase, skin problems and upper respiratory tract infections develop, and patients begin to lose weight. Persistent fever, fungal or repeated bacterial infections, and tuberculosis may follow.Besides, there are also other opportunistic infections and pathologic organisms which cause gastro intestinal diseases lading to chronic diarrhea.At the later stages of the disease, neurological manifestations can predominate and affect any part of nervous system [3].

More than 20 specific opportunistic infections have been associated with HIV infection [4] and patients usually experience co-infections

*Corresponding author: Mihiretu Alemayehu, School of Public Health, College of Health Sciences and Medicine, Wolaita Sodo University, Wolaita Sodo, PO Box138, Ethiopia; Tel: +251932017301; E-mail:mihiretua@gmail.com

Received January 17, 2017; Accepted February 07, 2017; Published February 14,2017

Citation: Alemayehu M, Yisehak Y, Alaro W, Alemayehu B (2017) Opportunistic Infections among HIVIAIDS Patients taking Ante-Retroviral Therapy at Tertiary Care Hospital in Wolaita Zone, Southern Ethiopia. J AIDS Clin Res 8: 665. doi: 10.4172/2155-6113.1000665

Copyright: (c) 2017 Alemayehu M, et al. This is an open-access article distributed under the terms of the Creative Commons Attribution License, which permits unrestricted use, distribution, and reproduction in any medium, provided the original author and source are credited. 
during the course of their illness [5]. HIV-related opportunistic infections are associated with significant morbidity and mortality and virtually none can be eradicated, necessitating life-long suppressive therapy after an acute episode. Prevention of such illness through primary prophylaxis is therefore compelling [6].The most common opportunistic infection is tuberculosis which affects one third of the world's people living with HIV/AIDS [7]. Herpes zoster and other mild opportunistic infections commonly occur at earlier stages of the disease, whereas life threatening infections such as CNS toxoplasmosis and cryptoccocal meningitis occur at late stages of the disease. Some others like recurrent bacterial pneumonia and tuberculosis may occur in early as well as late WHO stages of the disease.

According to world health organization (WHO) report the number of people living with HIV/AIDS has increased from 33.3 million in 2010 to 36.7 million in 2015 including 2.1 million newly infected people. Sub-Saharan African countries share $69 \%$ of global HIV burden [2].

In Ethiopia, the adult HIV prevalence of Ethiopia was estimated to be $1.1 \%$ in 2015 , where more than 691 thousand people are estimated to be living with the disease. It is the second leading cause of death in Ethiopia resulting in death of more than 26 thousand people in 2015. More than $90 \%$ of HIV/AIDS deaths are attributable to opportunistic infections and malignancies [8]. Even though nationally representative and comprehensive data regarding the magnitude of opportunistic infections lacks in Ethiopia, some regional studies has shown the prevalence ranging from $19.7 \%$ to $48 \%$ [9-11].

The type of opportunistic infection which affects people living with HIV/AIDS varies from region to region [12]. Therefore, for the strategies of in HIV/AIDS related morbidity and mortality to be reduced, identification of opportunistic infections, their frequency and distribution plays significant role. Hence, this research assessed the prevalence of opportunistic infections among people living with HIV/AIDS so that responsible stake holders and communities who have similar setting can utilize the findings of this research for further planning and implementation of combating HIV/AIDS and associated death.

\section{Methodology}

\section{Study design and area}

A facility based cross-sectional study was conducted from August 1-September 20, 2016 in Wolaita Sodo teaching and referral hospital. The hospital is found in Wolaita Sodo, southern Ethiopia which is located in $329 \mathrm{~km}$ south of Addis Ababa, the capital city of Ethiopia. The hospital gives service for Wolaita zone and other adjacent zones.

\section{Study population and sampling}

A facility based cross sectional study was conducted in Wolaita Sodo University teaching and referral hospital. In order to obtain adequate sample size, the source population of the study was adjusted to be all HIV/AIDS patients who visited ART clinic of the hospital within the past 5 years (September 1, 2012 to August 30, 2016) preceding the study. Record review of randomly selected patients was done to collect information regarding the study. Systematic sampling technique was used to select the clinical records from the registration books. The total number of clients who visited ART clinic during the specified time period was 3104 , while the total sample size needed to conduct the study was 377 . As a result, every eighth case record was selected to be included in the study after selecting the first case record by lottery method.
The total sample size needed for the study was obtained by using the formula to calculate sample size for single population proportion. Using $95 \%$ confidence interval, $5 \%$ precision, $33.3 \%$ prevalence of at least one opportunistic infection in Debremarkos referral hospital, Northern Ethiopia [9], and 10\% non-response rate, the total sample size was 377 .

\section{Data management and analysis}

A pre-tested checklist was used to collect data from records of selected patients who visited ART clinic of the hospital during the aforementioned time period. Data was checked for its completeness before the analysis was done. The checklist was adapted from guidelines and other similar studies. The data was entered and analyzed using SPSS version 20. Descriptive statistics (mean, standard deviation and percentages) were used to characterize study subjects.

\section{Quality control}

The completed cheek list was checked every day by supervisors and investigators for completeness and clarity. Training was given for data collectors and pre-test was done among 20 records before the actual data collection commenced.

\section{Ethical consideration}

The actual data collection was begun after Wolaita Sodo university college of Health Science and medicine, school of public health ethical review committee approved the study. Furthermore, the aim of the study, privacy and confidentiality of patients' records was clearly communicated and consent was received from the aforementioned hospital.

\section{Result}

\section{Socio-demography}

A total of 362 study participants were included in our study making 96\% response rate. Among them, 198 (54.7\%) were females and the rest 164 (45.3\%) were males. Most of the patients (46.1\%) were under the age group of 35-39 years. More than half (203 (56.1\%)) of the respondents were orthodox Christian religion followers while 218 (60.2\%) were living in a marital union. Around 228 (62.9\%) of study participants' educational level was above secondary school whereas 54 $(14.9 \%)$ and 51 (14\%) of them were self-employed and government workers, respectively (Table 1).

\section{Magnitude of opportunistic infections}

Fifty-seven $(15.7 \%)$ of study participants were classified under WHO clinical stage II, whereas 175 (48.3\%) and 130 (35.9\%) were classified under stage III and IV, respectively. Out of the 362 patients taking ART, 164 had at least one opportunistic infection making the overall magnitude to be $45.3 \%$. Only 25 (6.9\%) of them had double opportunistic co-infection while none of them had more than three co-infections. The most prevalent opportunistic infection was oral candidiasis followed by herpes zoster and pulmonary tuberculosis diagnosed among 64 (17.7\%), 56 (15.5\%) and 43 (11.9\%), respectively. The overall prevalence of tuberculosis (all forms of tuberculosis) among study participants was 70 (19.4\%), i.e. when considering all forms of tuberculosis together, it is the top prevalent opportunistic infection among study participants. Cryptoccocal meningitis, cryptosporidiosis and recurrent mycosis were the least commonly observed opportunistic infections accounting for $10(2.8 \%), 9(2.5 \%), 4(1.4 \%)$, respectively. AIDS defining illnesses such as CNS toxoplasmosis, cryptoccocal meningitis, 


\begin{tabular}{|c|c|c|}
\hline Variable & Frequency & Percent \\
\hline \multicolumn{3}{|l|}{ Sex } \\
\hline Male & 164 & 45.3 \\
\hline Female & 198 & 54.7 \\
\hline \multicolumn{3}{|l|}{ Age } \\
\hline$\leq 30$ & 40 & 11.1 \\
\hline $31-35$ & 83 & 22.9 \\
\hline $36-40$ & 167 & 46.1 \\
\hline Above 40 & 72 & 19.9 \\
\hline \multicolumn{3}{|l|}{ Marital status } \\
\hline Single & 63 & 17.4 \\
\hline Married & 218 & 60.2 \\
\hline Divorce/widowed & 81 & 22.4 \\
\hline \multicolumn{3}{|l|}{ Religion } \\
\hline Protestant & 128 & 35.4 \\
\hline Orthodox & 203 & 56.1 \\
\hline Muslim & 14 & 3.9 \\
\hline Others & 17 & 4.7 \\
\hline \multicolumn{3}{|l|}{ Ethnicity } \\
\hline Wolaita & 237 & 65.5 \\
\hline Others & 125 & 34.5 \\
\hline \multicolumn{3}{|l|}{ Education status } \\
\hline Can't read and write & 24 & 6.6 \\
\hline Primary* & 110 & 30.4 \\
\hline Secondary and above ${ }^{\star *}$ & 228 & 63 \\
\hline \multicolumn{3}{|l|}{ Occupation } \\
\hline Government employee & 51 & 14.1 \\
\hline Private employee & 54 & 14.9 \\
\hline Others $\mathbf{S}^{* * *}$ & 257 & 71 \\
\hline
\end{tabular}

* - Grade 1-8 **- Grade 9 and above ***- Daily laborer, merchant, housewife, etc

Table 1: Socio demographic characteristics of study participants, Opportunistic Infections among HIVIAIDS Patients taking ante-retroviral therapy at tertiary care hospital in Wolaita Zone, southern Ethiopia, 2016.
PCP (pneumocystis carini pneumonea) and cryptosporidiosis with diarrhea, which commonly manifest during the end stage of HIV/ AIDS, cumulatively account for 53 (14.6\%) of opportunistic infections among study participants (Figure 1).

\section{Discussion}

This study identified major opportunistic infections and their magnitude among people living with HIV/AIDS taking anti-retroviral therapy. The study found overall magnitude of opportunistic infection to be $45.3 \%$. this finding is in line with a study done in eastern Ethiopia in which $48 \%$ of study participants had opportunistic infection [10]. Similarly, it is consistent with $47.6 \%$ prevalence in Taiwan [13]. However, this finding is quite lower than $61.7 \%$ prevalence in Nigeria [14]. The discrepancy might be due to difference in characteristics of study participants that our study participants were solely those enrolled in ART, while the aforementioned study had included all sero positive patients irrespective of treatment status. Another study conducted in Nigeria revealed $22.4 \%$ prevalence of opportunistic infection [15]. Even though the study unit was the same as current study (taking antiretroviral therapy), nevertheless, the socioeconomic, nutritional and life style difference can result in variation of opportunistic infection prevalence. Our finding is also higher than a study done in Northern Ethiopia in which $19.7 \%$ of study subjects were found to be infected by one or more opportunistic infections [11]. The discrepancy might be the result of difference in study design. I.e. our study involved a fiveyear retrospective data while the above study confined to only threemonth duration.

Majority of study participants had one opportunistic infection while only $6.9 \%$ had a co-infection. Most common concomitant infections were Oral candidiasis, herpes zoster and pulmonary tuberculosis. This is in line with reports from Debremarkos, Eastern Ethiopia and Gondar [9-11].

In the current study, the most prevalent opportunistic infection

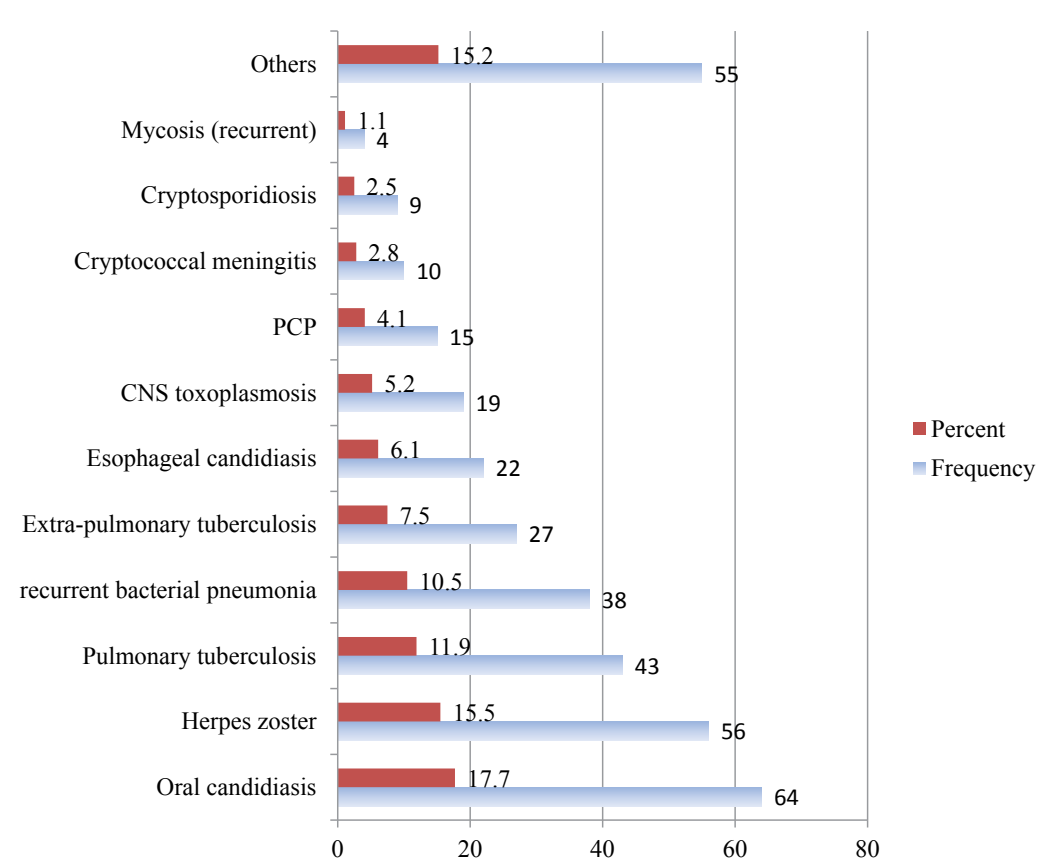

Figure 1: Prevalence of opportunistic infections among HIVIAIDS patients taking ante-retroviral therapy at tertiary care hospital in Wolaita Zone, southern Ethiopia, 2016. 
Citation: Alemayehu M, Yisehak Y, Alaro W, Alemayehu B (2017) Opportunistic Infections among HIVIAIDS Patients taking Ante-Retroviral Therapy at Tertiary Care Hospital in Wolaita Zone, Southern Ethiopia. J AIDS Clin Res 8: 665. doi: 10.4172/2155-6113.1000665

Page 4 of 4

was oral candidiasis (17.7\%). This is in agreement with a finding from Debremarkos, where oral candidiasis ranked the first among opportunistic infections [9]. Even though the prevalence of pulmonary and extra pulmonary tuberculosis ranked third and fifth with rate of $11.9 \%$ and $7.5 \%$, respectively, the combined prevalence of tuberculosis (pulmonary and extra pulmonary tuberculosis) was the top prevalent opportunistic infection among current study participants. This is in line with a report ofHabtamu $\mathrm{M}$ et al,in which $21.2 \%$ of study participants had any form of tuberculosis [10].

Next to oral candidiasis, herpes zoster is the third common opportunistic infection diagnosed among $15.5 \%$ of patients. This finding is consistent with a study from India where $14.7 \%$ of study subjects were diagnosed to be infected by herpes zoster [16]. AIDS defining illness which commonly manifest at late stage of the disease, were relatively less common among current study participants. Cryptoccocal meningitis, cryptosporidiosis and recurrent mycosis were the least commonly observed opportunistic infections accounting for $2.8 \%, 2.5 \%$, and $1.4 \%$ respectively. Cryptoccocal meningitis prevalence was slightly higher than a study from Debremarkos and Eastern Ethiopia in which $0.5 \%$ and $0.28 \%$ of study subjects had cryptoccocal meningitis, respectively $[9,10]$. Similarly, $5.2 \%$ prevalence of CNS toxoplasmosis is also relatively higher than $1.4 \%$ prevalence in Eastern Ethiopia [10]. Cumulative prevalence of AIDS defining illnesses, (CNS toxoplasmosis, Cryptoccocal meningitis, PCP and cryptosporidiosis with diarrhea) account for $14.6 \%$ of overall opportunistic infections. The relatively higher prevalence of AIDS defining opportunistic infections among current study participants might be the result of high number of patients classified under WHO stage IV. Accordingly, 35.9\% of study participants were classified under stage IV while $15.7 \%$ of study participants were classified under WHO clinical stage II and the rest $48.3 \%$ were classified under stage III.

\section{Conclusion and Recommendation}

This study revealed a high prevalence of opportunistic infection among HIV/AIDS patients taking anti-retroviral therapy. This indicates that the threatening burden of opportunistic infections couldn't be averted by sole provision of ante-retroviral therapy. The most common opportunistic infections were of tuberculosis (pulmonary and extrapulmonary), oral candidiasis and herpes zoster. Even though AIDS defining illnesses were relatively less prevalent, cumulatively they account for more than one sixth of overall opportunistic infections prevalence. Thus, in order to reduce the magnitude of the diseases, strategies beyond providing ante-retroviral therapy are required. In addition, researchers and other stake holders have to conduct longitudinal studies in order to identify risk factors for respective opportunistic infections.

\section{Ethical Approval and Consent to Participate}

The actual data collection was begun after Wolaita Sodo university college of Health Science and medicine approved the study. Furthermore, the aim of the study, privacy and confidentiality of patients' records was clearly communicated and consent was received from the aforementioned hospital

\section{Author's Contribution}

Yilma $\mathrm{Y}$ and Worku A: Conceived the study. Mihiretu A, Yilma Y, Worku A and Bereket A: Participated in the design of the study, performed statistical analysis, obtained ethical clearance and permission for the study, supervised data collection, interpreted the data and involved in drafting article. All authors read and approved the final manuscript.

\section{Authors' Information}

Mihiretu A is Master's of Public Health (MPH) in Reproductive Health specialty and works as lecturer in school of Public Health, College of Health Sciences and Medicine, Wolaita Sodo University, Ethiopia. Yilma Y and Worku A. are Public Health officers and work as health professional in Wolaita Zone, Southern Ethiopia. Bereket A. is a Master's of Science in Biomedical Science and works as lecturer in college of natural and computational science, Wolaita Sodo University, Ethiopia.

\section{Acknowledgement}

Wolaita Sodo University, Wolaita Sodo University teaching and referral hospital, ART clinic staffs deserve our special thank.

\section{References}

1. Guidelines For Management Of Opportunistic Infections And Anti-Retroviral Treatment In Adolescents And Adults In Ethiopia (2008) Federal HIVIAIDS Prevention And Control Office: Federal Ministry Of Health.

2. http://www.unaids.org/en/resources/documents/2016/Global-AIDSupdate-2016

3. http://www.aidsdatahub.org/hiv-related-opportunistic-diseases-unaidstechnical-update-unaids-1998

4. World Health Organization (2007) WHO case definitions of HIV for surveillance and revised clinical staging and immunological classification of HIV-related disease in adults and children.

5. Saag MS (1994) Natural history of HIV-1 Disease. Textbook of AIDS medicine: Williams \& Wilkins, Baltimore 45.

6. Gangadhara T, Ramesh K (2014) Opportunistic infections among HIV patients attending tertiary care hospital, Karnataka, India. International Journal of Current Microbiology and Applied Sciences 3: 824-829.

7. Sharma SK, Mohan A, Kadhiravan T (2005) HIV-TB co-infection: Epidemiology, diagnosis and management. Indian J Med Res, pp: 550-567.

8. http://www.etharc.org/index.php/resources/healthstat/hivaids-estimates-andprojections-in-ethiopia-2011-2016

9. Moges NA, Kassa GM (2014) Prevalence of opportunistic infections and associated factors among HIV positive patients taking antiretroviral therapy in Debremarkos referral hospital, Northwest Ethiopia. J AIDS Clin Res 5: 301.

10. Habtamu M, Fitsum W, Zelalem T (2015) Magnitude of opportunistic infections and associated factors in HIV-infected adults on antiretroviral therapy in eastern Ethiopia. HIV AIDS (Auckl) 7:137-144.

11. Ayyagari A, Sharma AK, Prasad KN, Dhole TN, Kishore J, et al. (1999) Spectrum of opportunistic infection in Human immunodeficiency virus (HIV) infected cases 339 in tertiary care hospital. Ind J Med Microbiol 17: 78-80.

12. Sun HY, Chen MY, Hsieh SM (2006) Changes in the clinical spectrum of opportunistic illnesses in persons with HIV infection in Taiwan in the era of highly active antiretroviral therapy. Jpn J Infect Dis 59: 311-316.

13. Saidu AS, Bunza MD, Abubakar U, Adamu T, Ladan M, et al. (2009) A survey of opportunistic infections in HIV seropositive patients attending major hospitals of Kebbi state, Nigeria. Bayero Journal of Pure and Applied Sciences 2: 70-74.

14. Iroezindu MO, Ofondu EO, HauslerH, VanWyk B (2013) Prevalence and risk factors for opportunistic infections in hiv patients receiving antiretroviral therapy in a resource-limited setting in Nigeria. J AIDS Clinic Res S3: 002.

15. Damtie D, Yismaw G, Woldeyohannes D, Anagaw B (2013) Common opportunistic infections and their CD4 cell correlates among HIV-infected patients attending at antiretroviral therapy clinic of Gondar University Hospital, Northwest Ethiopia. BMC Res Notes 6: 534.

16. Wood R, Maartens G, Lombard CJ (2000) Risk factors for developing tuberculosis in HIV-1-infected adults from communities with a low or very high incidence of tuberculosis. J Acquir Immune Defic Syndr 23: 75-80. 